\title{
Network Analysis and Visualisation ${ }^{\star}$
}

\author{
Seok-Hee Hong \\ National ICT Australia, School of Information Technologies, \\ University of Sydney, Australia \\ shhong@it.usyd.edu.au
}

\begin{abstract}
A workshop on Network Analysis and Visualisation was held on September 11, 2005 in Limerick Ireland, in conjunction with 2005 Graph Drawing conference. This report review the background, progress and results of the Workshop.
\end{abstract}

\section{Motivation}

Recent technological advances produce many large and complex network models in many domains. Examples include web-graphs, biological networks and social networks.

Visualisation can be an effective tool for the understanding of such networks. Good visualisation reveals the hidden structure of the networks and amplifies human understanding, thus leading to new insights, new findings and possible predictions for the future.

Analysis methods are available for these networks. However, analysis tools for networks are not useful without visualisation, and visualisation tools are not useful unless they are linked to analysis.

This workshop aimed to gather researchers interested in the analysis and visualisation of large and complex networks. More specifically, the workshop had the objectives identifying new research opportunities in network analysis and visualisation, and encouraging collaborative solutions in this area.

\section{Workshop Overview}

The workshop was chaired by Seok-Hee Hong (National ICT Australia and University of Sydney, Australia), and organised with Dorothea Wagner (University of Karlsruhe, Germany) and Michael Forster (National ICT Australia, Australia).

It had the following 35 participants from 10 countries: Radoslav Andreev (University of Limerick, Ireland), Vladimir Batagelj (University of Ljubljana, Slovenia), Michael Baur (University of Karlsruhe, Germany),Elena Besussi (Uni-

\footnotetext{
* This workshop was supported by NICTA (National ICT Australia), funded by the Australian Government's Backing Australia's Ability initiative, in part through the Australian Research Council.
} 
versity College London, UK), Ulrik Brandes (University of Konstanz, Germany), Stina Bridgeman (Hobart and William Smith Colleges, US), Ali Civril (Rensselaer Polytechnic Institute, US), Walter Didimo (University of Perugia, Italy), Tim Dwyer (Monash University, Australia), Jean-Daniel Fekete (INRIA Futurs, France), Michael Forster (National ICT Australia, Australia), Marco Gaertler (University of Karlsruhe, Germany), Francesco Giordano (University of Perugia, Italy), Luca Grilli (University of Perugia, Italy), Martin Harrigan (University of Limerick, Ireland), Patrick Healy (University of Limerick, Ireland), Nathalie Henry (INRIA Futurs, France), Joshua Ho (University of Sydney, Australia), Seok-Hee Hong (National ICT Australia and University of Sydney, Australia), Tony Huang (National ICT Australia and University of Sydney, Australia), Dirk Koschtzki (IPK Gatersleben, Germany), Karol Lynch (University of Limerick, Ireland), Anila Mjeda (Waterford Institute of Technology, Ireland), Nikola Nikolov (University of Limerick, Ireland), Andreas Noack (University of Cottbus, Germany), Aaron Quigley (University College Dublin, Ireland), Aimal Rextin (University of Limerick, Ireland), Falk Schreiber (IPK Gatersleben, Germany), Matthew Suderman (McGill University, Canada), Antonios Symvonis (National Technical University of Athens, Greece), Alexandre Tarassov (University of Limerick, Ireland), Ioannis Tollis (University of Crete, Greece), Francesco Trotta (University of Perugia, Italy), Dorothea Wagner (University of Karlsruhe, Germany), Michael Wybrow (Monash University, Australia).

\section{Invited Talks}

The workshop had two invited talks for one hour each as follows.

\section{- Vladimir Batagelj: Some Visualization Challenges from Social Network Analysis}

Network $=$ Graph + Data. The data can be measured or computed/derived from the network. In traditional graph drawing the goal was to produce the best layout of given graph. SNA (Social Network Analysis) is a part of data analysis. Its goal is to get insight into the structure and characteristics of given network. There is no single answer - usually we are trying to find interesting facts about the network and present them to the users. The visualization is a tool for network exploration and for presentation of the final results. This process requires combination of analysis and visualization techniques.

SNA deals also with multi-relational, temporal and often large networks. The standard sheet of paper paradigm is often not appropriate for the amount of information in such networks. It was addressed that one should develop dynamic interactive layouts, introduce new visualization elements to represent typical network substructures, and add some artistic touch to final displays. The slides of the talk are available at: http://vlado.fmf.uni-lj.si/pub/networks/doc/mix/GDaSNA.pdf. 


\section{- Ulrik Brandes: Visualization of Dynamic Social Networks}

Social network visualization is a specific area in the field of information visualization. Models of social structures typically consist of some type of graph data (where variations are with respect to directedness, multiple edges, bipartiteness, etc.) together with an arbitrary set of attributes. While there are many unsolved problems in visualizing social structures, dynamic changes of the graph and/or its attributes introduce an additional dimension of com-

plexity. In this talk, an attempt was made to point out directions relevant to social network analysts and open problems interesting to the graph drawing community. Several examples from recent social network studies were used for illustration.

\section{Contributed Talks}

The workshop had four contributed talks for 30 minutes each as follows.

- Falk Schreiber: Visual Network Analysis for Systems Biology Systems biology is a new field in biology that aims at the understanding of complex biological systems, such as a complete cell. It has emerged in the light of the availability of modern high-throughput technologies, which result in huge amounts of molecular data regarding life processes. This data is often related to, or even structured in, the form of biological networks such as metabolic, protein interaction and gene regulatory networks. Visual data exploration methods help scientists to extract information out of the data and thus are very useful for building sophisticated research tools. This presentation gave a brief introduction into systems biology and molecular biological processes. It discussed examples of the analysis of fundamental biological networks and their user-friendly visualisation. These examples range widely from visualising experimental data in the context of the underlying biological networks to structural analysis and subsequent visualisation of biological networks based on motifs, clustering and centralities. Finally new directions and questions in visualising these large and complex networks were considered.

- Nathalie Henry: Matrices for Visualizing Social Networks Visualizing and interacting with large social networks is a challenging task. Usually, social networks are represented as node-link diagrams. These representations are intuitive and effective for filtered or aggregated social networks. In this talk it was proposed to use matrix-based representations to interact and manipulate social networks prior to node-link diagram visualization. Adjacency matrix representations are easier to interact with in term of navigation and computation such as filtering, clustering or aggregation. These representations may offer the user a good overview of large and dense social networks. The main issue when dealing with matrices is their rows and columns ordering. Ordering is a key to understand a matrix or more generaly a table. An overview of existing methods to reorder tables was presented 
with explanation of how automatic methods work in detail and how to apply these methods to adjacency matrices. Finally focus on matrix readability with experimental work was presented.

- Tim Dwyer: New Techniques for Visualisation of Large and Complex Directed Graphs

To date, the famous Sugiyama algorithm has been the method of choice for drawing directed graphs. Prime examples are drawings of metabolic pathways and UML diagrams. However, when used to draw large and complex directed graphs - with hundreds or thousands of nodes and a high density of edges - the method does not produce very readable diagrams. This talk argued that a method based on techniques from the field of multi-dimensional scaling coupled with some custom constrained optimisation techniques scales much better to the visualisation of large and complex directed graphs.

- Michael Wybrow: Visualisation of Constraint-Based Relationships in Graphs and Diagrams

Constraint-based relationships in diagrams are permanent placement relationships such as alignment, distribution, left-of, right-of, pinning, etc. They are maintained by the diagram editor as objects are added, removed or moved around. It is clear there should be an on-screen representation of such objects, but in practice these tend to quickly clutter the page and make comprehension of the graph and the constraint relationships themselves difficult. This talk demonstrated the problem and offered for discussion some possible solutions using proximity, transparency, time, and knowledge of active/broken constraints.

\section{Discussion}

The following open problems were suggested and discussed by the participants:

- Ulrik Brandes: Adapt Sugiyama paradigm (i.e. level assignment, crossing reduction, $\mathrm{x}$-coordinates) with partially given $\mathrm{y}$-coordinates

- Vladimir Batagelj:

- New drawing styles and conventions

- Interactive network layout

- Matrix Layout

- Generalized blockmodeling

- Visualisation with additional graphical elements

- Visualisation of multi-relational and dynamic networks

- Dense directed network layout

- Jean-Daniel Fekete: Evaluation and usability studies of other network visualisation methods (besides node-link diagrams) 